
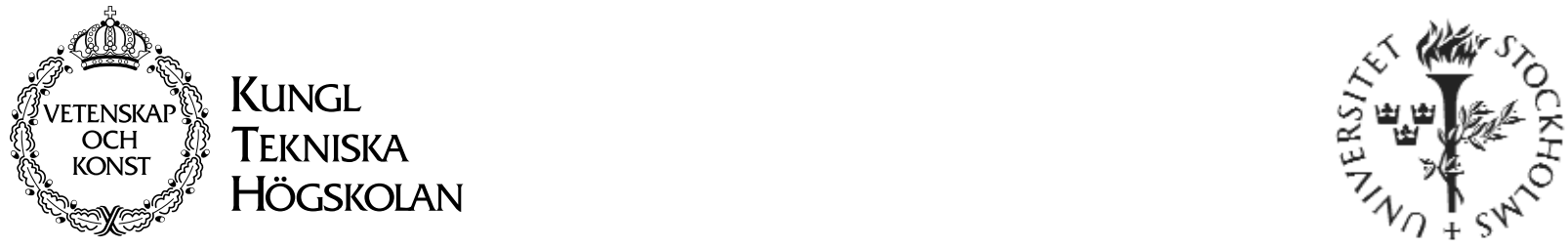

CID-137 • ISSN 1403-0721 • Department of Numerical Analysis and Computer Science $\bullet$ KTH

\title{
A System for Exploring Open Issues in VR-based Education.
}

Gustav Taxén and Ambjörn Naeve 


\section{Gustav Taxén and Ambjörn Naeve}

A System for Exploring Open Issues in VR-based Education Report number: CID-137

ISSN number: ISSN 1403 - 0721 (print) 1403 - 073 X (Web/PDF)

Publication date: August 2001

E-mail of author: gustavt@ nada.kth.se, amb@ nada.kth.se

\section{Reports can be ordered from:}

CID, Centre for User Oriented IT Design

NADA, Deptartment of Numerical Analysis and Computer Science

KTH (Royal Institute of Technology)

SE- 10044 Stockhom, Sweden

Telephone: $+46(0) 87909100$

Fax: + 46 (0)8 7909099

E-mail: cid@nada.kth.se

URL: http://cid.nada.kth.se 


\title{
A System for Exploring Open Issues in VR-based Education
}

\author{
Gustav Taxén Ambjörn Naeve \\ Center for user-oriented IT design \\ The Royal Institute of Technology \\ Lindstedtsvägen 5 \\ S-100 44 Stockholm, Sweden \\ +4687909277 \\ [gustavt | amb]@ nada.kth.se
}

\begin{abstract}
Virtual Reality (VR) has been shown to be an effective way of teaching difficult concepts to students. However, a number of important questions related to immersion, collaboration and realism remain to be answered before truly efficient virtual learning environments can be designed. We present CyberMath, an avatar-based shared virtual environment for mathematics education that allows further study of these issues. In addition, CyberMath is easily integrated into school environments and can be used to teach a wide range of mathematical subjects.
\end{abstract}

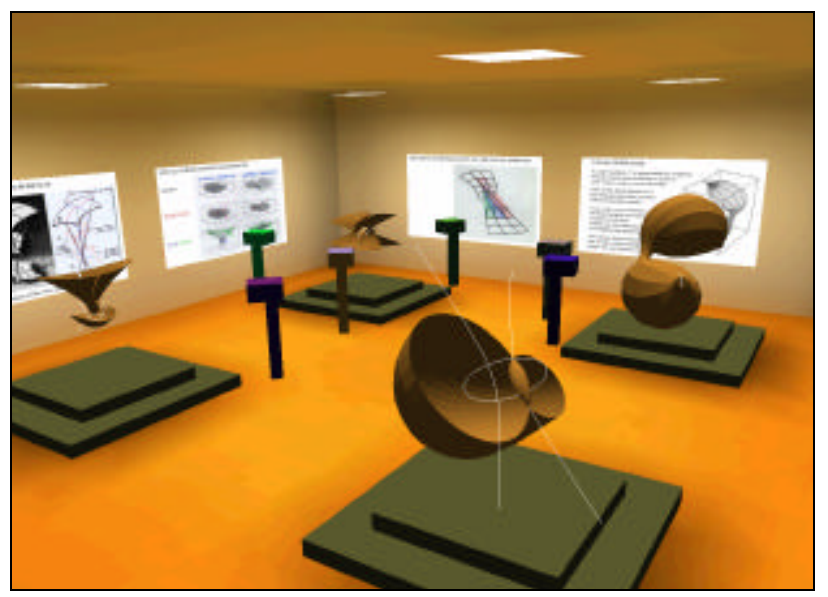

Figure 1. A CyberMath exhibition on focal surfaces.

\section{INTRODUCTION}

Virtual Reality systems have the potential to allow students to discover and experience objects and phenomena in ways that they cannot do in real life. Since the early 90s, a large number of educational VR applications have been developed. These include tools for teaching students about physics [6], algebra [1], color science [16], cultural heritage objects [17] and the greenhouse effect [10].
There is convincing evidence that students can learn from educational VR systems [19]. However, a number of unresolved issues regarding the efficiency of such systems still remain. These include:

Immersive vs. non-immersive VR. Several different authors have shown that immersive VR, where the user is in a CAVE or wears a head-mounted display, can be more efficient for learning than monitor-based desktop VR [4]. However, current immersive VR systems are expensive, fragile, and can be cumbersome to use. These drawbacks make them hard to integrate into school environments. On the other hand, desktop VR systems can often run on standard PC hardware, equipment that is increasingly common in classrooms today. Also, students using desktop VR systems are less likely to experience motion sickness and fatigue, factors that are known to inhibit learning [7]. It is unclear whether the advantages of desktop VR systems can make up for their lack of immersion.

Collaboration in educational VR systems. A number of different initial studies suggest that collaboration between students in virtual environments have a positive effect on learning [10][14][2][12]. However, little is known about how the presence of a teacher influences learning in VR applications. It is likely that students will benefit from teacher guidance, but it is also possible that a system that allows the teacher to take a more active role within the virtual environment would have a positive effect.

Avatar-based multi-user virtual environments often induce the formation of user communities. The increased level of anonymity and "safety" in such communities may encourage users that usually avoid experiential learning situations to participate in educational activities [5]. However, it can be more difficult to avoid digression in discussions when the participants are anonymous than when they are known to each other [11]. There are few available guidelines for handling large-scale participation in educational VR systems. 
Visual realism in educational VR systems. A number of different studies have shown that visual realism in VR applications must be used with care [18]. It is not certain that an increased level of realism will improve learning since it may distract a student from focusing on the key concepts that is to be learned. However, the motivational value of excessive visual realism is very high, something that the motion picture and computer games industries have been taking advantage of for decades. How to use realism in order to highlight key relations and concepts in educational VR applications is still an open question.

This paper presents CyberMath, a system in which all of these issues can be explored. To our knowledge, no previous educational VR system has all the features necessary for such studies. In addition, CyberMath is built to support the teaching of many mathematical subjects, ranging from elementary school content to post-graduate content. Our system also supports a variety of teaching styles, including teacher lecturing and student-initiated exploration.

\section{SYSTEM DESCRIPTION}

CyberMath is an avatar-based shared virtual environment that is built on top of DIVE [3]. DIVE has the ability to display interactive three-dimensional graphics as well as to distribute live audio between standard desktop PCs. It also supports a number of other hardware configurations, ranging from head-mounted displays to CAVE environments. It is possible to allow different users to access the same virtual environment from workstations with different hardware configurations. These features make it easy to integrate DIVE applications in schools and also allow us to study how different levels of immersion influence the learning process.

Many students have considerable difficulty appreciating the relevance of mathematics. We believe that an informal and fun milieu aids in motivating such students and also encourages the formation of user communities. Therefore, we have chosen to build CyberMath as an exploratorium that contains a number of exhibition areas (figure 1). This allows teachers to guide students through the exhibitions but students can also visit CyberMath at their leisure, alone or together with others. For additional flexibility, we have added a lecture hall where standard PowerPoint presentations can be shown. Furthermore, since DIVE can distribute information across multiple local area networks, users from different physical locations can visit the exploratorium simultaneously.

Multiple users can simultaneously manipulate an exhibition object in CyberMath. In order to reduce confusion, it is important to make explicit the presence of users in the virtual environment. In addition, we believe that visualizing user presence increases the potential for user-to-user collaboration and interaction. For simplicity, we have chosen to let the standard DIVE avatars represent
CyberMath visitors, but we are currently experimenting with alternative avatar designs. The way users control their avatars in CyberMath is similar to many popular computer games. Since many students are familiar with these games, our hope is that this will shorten the time required to master the controls.

When a user points to an object in the environment using the computer mouse, his/her avatar will indicate this through a "laser pointer" - a red line from the eye of the avatar through the indicated point on the object. Each avatar also has a sound indicator that is activated when its corresponding user speaks into the computer microphone. Exhibited objects can be rotated and translated by using the computer mouse. Action buttons situated next to interactive exhibitions control animations and visual representation of the objects in the exhibit.

All objects in CyberMath, including the user avatars, can be visualized at a number of different levels of realism, ranging from uniformly colored surfaces to radiosity lighting. This makes it possible to investigate how realism affects learning in virtual environments.

DIVE supports rapid prototyping through Tcl/Tk scripts. We have complemented this support with a Mathematicato-DIVE conversion utility that can be used to convert standard three-dimensional Mathematica objects and animations to the DIVE file format. It is then straightforward to add Tcl/Tk code to turn the converted Mathematica objects into interactive CyberMath exhibitions. This makes it possible to support rapidturnaround teacher-driven development of new CyberMath exhibitions in the same fashion as in the QuickWorlds project [13]. The next step is to develop an exhibition construction tool that will allow teachers without Tcl/Tk knowledge to create their own exhibitions.

It is also possible to associate URLs with CyberMath exhibition objects. When a user clicks on such an object, its URL is opened in a WWW browser. This makes it easy to offer additional information about the exhibited objects (such as mathematical formulae and links to other relevant WWW pages).

DIVE has the ability to log all interactions between avatars and objects. Together with standard audio and video recording equipment, this provides a platform for assessment of learning in CyberMath.

A number of example exhibition areas in the exploratorium have been completed. These include:

Interactive transformations. In this exhibit, users can explore the effect of any $\mathbf{R}^{3} \rightarrow \mathbf{R}^{3}$ transformation on different mathematical entities such as points, lines, planes and spheres. The user can interactively manipulate the entities and immediately see the results of the transformation, either in a separate coordinate frame or in the same coordinate frame as the untransformed surface 
(figure 2). This makes it possible to explore transformations in a new way and get an intuitive sense for how a specific transformation works. We believe that this increases the cognitive contact with the mathematical ideas behind the transformation formulae.

Generalized cylinders. This exhibition illustrates how to increase the number of degrees of freedom in revolution surfaces through the use of differential geometry [15]. In particular, it is shown how to construct an orthogonal net across the surfaces for texture mapping. The exhibition includes a number of three-dimensional animations and wall posters. Differential geometry is usually taught at the post-graduate level (if at all). However, our initial usability tests indicate that CyberMath makes it possible to effectively introduce these concepts to undergraduate students.

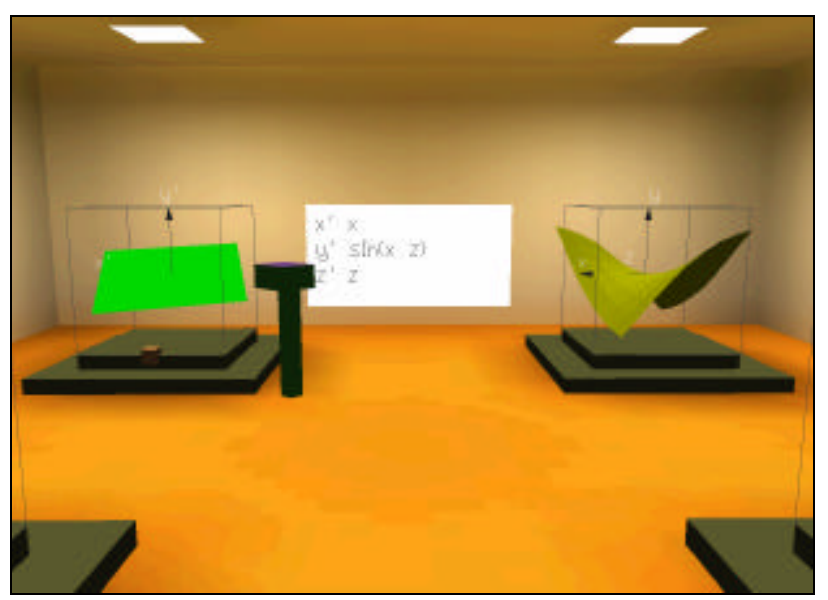

Figure 2. The interactive transformations exhibition. The user is manipulating the green plane in the domain on the left and the corresponding transformed surface appears in yellow on the right. The transformation is displayed on the wall between the two coordinate systems.

\section{USABILITY TESTING}

We have completed two initial usability tests, one small test at our lab with three users and one larger test with fourteen users. In both tests, the students were undergraduates at different universities in the Stockholm region. A mathematics teacher from the Royal Institute of Technology (that is familiar with CyberMath) guided the students through the generalized cylinders exhibition hall. The teacher was in a separate physical location and all students were sitting at different workstations in one room. After the guided tour, the students answered a 1-to-5 rating Likert-scale questionnaire. The questions were divided into four themes:
1. Effectiveness of the human/computer interface (navigation, sound quality, orientation of avatars, etc.).

2. Perceived level of immersion and awareness of other users in the virtual environment.

3. Level of collaboration (teacher-student and studentstudent).

4. Transfer of content, feasibility of CyberMath as teaching tool.

The average ratings for these themes were 3.23, 3.49, 3.35 and 4.10, respectively. These results are hardly conclusive. Nevertheless, they suggest that even though improvements in user interface and environment design are necessary, CyberMath has the potential of becoming a powerful tool for teaching mathematics.

We are planning a larger deployment of CyberMath at the Royal Institute of Technology and a series of new usability tests. These tests will focus on three main areas:

- Immersion: To what extent do different levels of immersion (desktop monitor, wall projection, headmounted display, CAVE) influence the long-term retainment of knowledge acquired through virtual environments?

- Collaboration and teaching strategies: How does the possibility of large-scale participation influence the teaching and learning processes? To what extent must teachers adapt their teaching style in collaborative virtual environments?

- Realism: Can the increased motivational value of a realistic environment compensate for the lack of immersion in desktop-based systems? Can we produce a set of guidelines for using visual realism in virtual environments for education?

Our hope is that these tests will produce new insights into how to design efficient VR systems for education. We are also planning to build a number of new exhibition areas, including one that presents elementary three-dimensional geometry and one that introduces geometric algebra [8]. We will use results from research on awareness and accommodation in virtual environments to further guide the design of these exhibition areas [9].

\section{REFERENCES}

1. Bricken, W. Spatial Representation of Elementary Algebra. In Proceedings of the 1992 IEEE Workshop on Visual Languages, 55-62.

2. Brna, P., Aspin, R. Collaboration in a Virtual World: Support for Conceptual Learning? In Proceedings of the IFIP WG 3.3 Working Conference "HumanComputer Interaction and Educational Tools”, 113123. 
3. Carlsson, C., Hagsand, O. DIVE - A Multi User Virtual Reality System, In Proceedings of IEEE VRAIS '93, 394-400.

4. Cronin, P. Report on the Applications of Virtual Reality Technology to Education. HRHC, University of Edinburgh, February

1997. http://www.cogsci.ed.ac.uk/ paulus/vr.html

5. Dede, C. The Evolution of Constructivist Learning Environments: Immersion in Distributed, Virtual Worlds. In Educational Technology, 35 (5), 1995, 4652.

6. Dede, C., Salzman, M. C., Loftin, R. B. ScienceSpace: Virtual Realities for Learning Complex and Abstract Scientific Concepts. In Proceedings of IEEE VRAIS '96, 246-252.

7. Dede, C., Salzman, M., Loftin, R. B., Ash, K. Using Virtual Reality Technology to Convey Abstract Scientific Concepts. In Jacobson, M. J., Kozma, R. B. (Ed.), Learning the Sciences of the $21^{\text {st }}$ Century: Research, Design, and Implementing Advanced Technology Learning Environments. Lawrence Erlbaum, 1997.

8. Doran, C., Dorst, L., Hestenes, D., Lasenby, J., Mann, S., Naeve, A., Rockwood, A. Geometric Algebra: New Foundations, New Insights, ACM SIGGRAPH '00 Course Notes.

9. Hedman, A., Lenman, S. Orientation vs. Accommodation - New Requirements for the HCI of Digital Communities. In Proceedings of HCII '99, 457461.

10.Jackson, R. L. Peer Collaboration and Virtual Environments: A Preliminary Investigation of MultiParticipant Virtual Reality Applied in Science Education. In Proceedings of the ACM 1999 Symposium on Applied Computing, 121-125.
11.Jin, Q., Yano, Y. Design Issues and Experiences from Having Lessons in Text-Based Social Virtual Reality Environments. In Proceedings of the 1997 IEEE International Conference on Computational Cybernetics and Simulation, vol. 2, 1418-1423.

12.Johnson, A., Roussos, M., Leigh, J., Vasilakis, C., Barnes, C., Moher, T. The NICE Project: Learning Together in a Virtual World. In Proceedings of IEEE VRAIS '98, 176-183.

13.Johnson, A., Moher, T., Leigh, J., Lin, Y-J. QuickWorlds: Teacher-Driven VR Worlds in an Elementary School Curriculum. In Proceedings of ACM SIGGRAPH 'OO Educators Program, 60-63.

14. Moher, T., Johnson, A., Ohlsson, S., Gillingham, M. Bridging Strategies for VR-Based Learning. In Proceedings of ACM CHI '99, 536-543.

15. Naeve, A., Eklundh, J. O. Representing Generalized Cylinders. In Proceedings of the 1995 Europe China Workshop on Geometric Modeling and Invariants for Computer Vision, 63-70.

16.Stone, P. A., Meier, B. J., Miller, T. S., Simpson, R. M. Interaction in an IVR Museum of Color. In Proceedings of ACM SIGGRAPH 'OO Educators Program, 42-44.

17. Terashima, N. Experiment of Virtual Space Distance Education System Using the Objects of Cultural Heritage. In Proceedings of the 1999 IEEE International Conference on Multimedia Computing and Systems, vol. 2, 153-157.

18. Wickens, C. D. Virtual Reality and Education. In Proceedings of the 1992 IEEE International Conference on Systems, Man and Cybernetics, vol. 1, 842-847.

19. Winn, W. The Impact of Three-Dimensional Immersive Virtual Environments on Modern Pedagogy. University of Washington, HITL, Report No. R-97-15, 1997. 